\title{
Creating Effective Self-Management for Older Adults with Type 2 Diabetes and Cognitive Impairment
}

\author{
Cameron J. Camp ${ }^{1 *}$, Kathleen Fox ${ }^{1}$, Michael J. Skrajner ${ }^{2}$, Vincent Antenucci ${ }^{1}$, \\ Jessica Haberman ${ }^{1,3}$ \\ ${ }^{1}$ Center for Applied Research, Solon, USA \\ ${ }^{2}$ Hearthstone Alzheimer's Care, Woburn, USA \\ ${ }^{3}$ Cleveland State University, Cleveland, USA \\ Email: ${ }^{*}$ Cameron@cen4ard.com
}

Received 10 February 2015; accepted 26 February 2015; published 3 March 2015

Copyright (C) 2015 by authors and Scientific Research Publishing Inc.

This work is licensed under the Creative Commons Attribution International License (CC BY). http://creativecommons.org/licenses/by/4.0/

(c) (i) Open Access

\section{Abstract}

The primary objective of the study was to determine whether a distanced-based educational intervention would result in positive health outcomes for persons with both DM and cognitive impairment. Older adults with Type 2 diabetes (Diabetes Mellitus-DM) who also have cognitive impairment such as Mild Cognitive Impairment (MCI) or early stage dementia are both challenged and at risk when attempting to live independently. The ability to effectively monitor blood glucose levels and diet and exercise regimens often is severely constrained by the combination of DM and the presence of Mild Cognitive Impairment (MCI) or early stage dementia. We describe an exploratory study funded by the National Institute of Diabetes Digestive and Kidney Diseases (NIDDK) in which Certified Diabetic Educators (CDEs) were linked with 40 older adult with DM and cognitive impairment using iPads and the internet. CDEs presented personalized education sessions to participants, and 18 of the participants also received a cognitive intervention called Spaced Retrieval (SR), which is designed to train the effective use of strategies to enhance medication compliance and reach other goals. Blood glucose and cholesterol measures were assessed at baseline and at 2-, 4-, and 6-month post intervention. Hemoglobin A1c (HbA1c) levels initially declined from baseline after treatment but returned to baseline levels after 6 months. For low-density lipoprotein (LDL) cholesterol, a significant interaction effect was found for the Group $\times$ Time interaction. LDL levels increased from baseline after treatment for the control group, but showed decline after baseline in the SR group. Goals that were initially learned were retained, in general, at short-term follow-up, and self-efficacy increased significantly after training. Results show the need for follow-up and support after initial treatment, as well as the need to see if the effects produced by SR can be replicated and sustained with continued contact.

\footnotetext{
${ }^{*}$ Corresponding author.
}

How to cite this paper: Camp, C.J., Fox, K., Skrajner, M.J., Antenucci, V. and Haberman, J. (2015) Creating Effective SelfManagement for Older Adults with Type 2 Diabetes and Cognitive Impairment. Advances in Aging Research, 4, 33-41. 


\section{Keywords}

\section{Type 2 Diabetes, Cognitive Impairment, Distance-Based Intervention, Educational Intervention, Spaced-Retrieval}

\section{Introduction}

Obesity is epidemic in the United States. In 2012, 38\% of females and 32\% of men aged 60 and over were obese [1]. Obesity, in turn, is a risk factor for Type 2 Diabetes. Among those age 65 years and older, 11.8 million, or 25.9\%, had diabetes in 2012 [2]. Diabetes was the seventh leading cause of death based on US death certificates in 2010 and, overall, the risk for death among people with diabetes is about twice that of people of similar age but without diabetes [2] [3] (Centers for Disease Control and Prevention, 2014; National Diabetes Fact Sheet, 2011).

Management of diabetes is complex, involving maintaining appropriate: weight, diet, exercise regimen, monitoring of blood sugar levels, and medication regimens. In older adults, management of co-morbidities (hypercholesterolemia, high blood pressure, etc.) can further add to the complexity and challenge of managing diabetes. Untreated or poorly managed diabetes can lead to blindness, amputations, organ failure, and death. With the impending influx of the boomer cohort into later adulthood, this poses a serious and immediate public health concern [1] [4] [5].

In addition, research suggests that the chronic stress of care giving, especially among older adults, may lead to conditions such as metabolic syndrome (a combination of abdominal obesity, high blood pressure, high blood glucose, and high cholesterol that is often associated with developing diabetes and heart disease) [6]-[9]. Metabolic syndrome, in turn, may have detrimental effects on health [10]. Thus, stressors common to older adults can be contributing factors in the development of diabetes and other co-morbid conditions.

Furthermore, diabetes is a known risk factor for impaired cognition and dementia. Epidemiological studies have shown that persons with Type 2 diabetes have a twofold increased risk of developing either Alzheimer's Disease or vascular dementia, with strong interaction of other factors such as hypertension, dyslipidemia, and apolipoprotein E phenotype [11]. Cognitive dysfunction includes memory and executive deficits, with likely roles for both vascular and non-vascular factors. The association between Type 2 diabetes and lower cognitive function, as well as greater cognitive decline, is supported by other investigators [12] [13]. For example, Haan [14] found that Type 2 diabetics with issues involving blood pressure showed a significantly greater rate of cognitive decline than nondiabetics.

For the increasing number of persons with both Type 2 diabetes and cognitive impairment, especially outpatients who may have limited capability and/or assistance in maintaining medical regimens, diabetes can be an extremely dangerous condition. In turn, impaired cognition can exacerbate the effects of Type 2 Diabetes and can hinder its management. Older adults with Type 2 Diabetes and even Mild Cognitive Impairment (MCI), especially those who are attempting to live independently, can be extremely challenged when attempting to manage diabetes. Again, presence of co-morbidities adds further impediments to these attempts.

Depression is also associated with poor diabetes self-management. This is an important consideration, because as many as $33 \%$ of individuals with diabetes have depression at a level that impairs functioning and quality of life [15]. Research has shown that depression is associated with less physical activity, unhealthy diet, and lower adherence to oral hypoglycemic, antihypertensive, and lipid-lowering medications [16]. Depressed persons with DM also have a greater probability of having any emergency department, primary care, specialty care, medical inpatient, and mental health costs; and among health care users, they have higher primary, ambulatory, and total health care costs [17].

In 2009 fully 29\% of Medicare recipients with Alzheimer's Disease (AD) or other dementias had diabetes. The average per-person payment by Medicare to beneficiaries Age 65 and older with diabetes and AD or other dementias is higher than for those with diabetes and no $\mathrm{AD} /$ dementia for the following services areas: hospital care, physician care, skilled nursing care, and home health services. In total, Medicare spends nearly twice the amount annually on persons who have both $\mathrm{AD} /$ dementia and diabetes when compared to those with diabetes and no AD/dementia diagnosis: \$24,776 vs. \$13,395 [10]. 
Among Medicare beneficiaries who reported having been diagnosed with AD or other dementias in the Medicare Current Beneficiary Survey, 42 percent lived in a nursing home or other facility that provided residential support (e.g., assisted living facility), 43 percent lived in the community with another person, and the remaining 15 percent lived alone in the community. Thus, among those who lived in the community, one out of four lived alone [10]. An estimated 5.2 million Americans had AD in 2014, of which 60 - 70 percent (3.12 - 3.64 million) lived in the community. Of these community dwelling persons, 75 percent ( $2.34-2.73$ million) lived with someone else, while 25 percent (780,000 - 910,000) were living alone [18].

Although many people with dementia are able to perform basic aspects of self-care, people with dementia and who live alone are at increased risk of inadequate self-care, including malnutrition, untreated medical conditions, inadequate clothing or housing, poor hygiene, and increased need for emergency medical services. The issue of inadequate self-care is compounded by the observation that many people who are unable to care for themselves refuse help [10]. This can be driven by the fear that acknowledging need for assistance will lead to loss of independence and ultimate placement in a long-term care setting such as a nursing home.

To summarize, the rapid increase in obesity in the US population, especially among older adults, has been related to a cascade of health problems, in particular an increase in Type 2 diabetes. Management of diabetes is complex, especially in the presence of co-morbidities commonly seen in older adults. Failure to adequately manage diabetes can lead to severe medical complication, and even death. Stressors such as those common in older adult caregivers can be contributing factors to development of diabetes and related co-morbidities. In addition, diabetes often occurs in the presence of cognitive impairment, which in turn can further challenge attempts to manage this chronic condition. Co-occurrence of depression can also negatively impact one's ability to selfmanage. Many older adults with cognitive impairment associated with dementia currently are attempting to live independently and alone in the community. These persons are at extreme risk, yet often are hard to reach and may refuse help. A pressing public health need therefore exists to provide support and intervention to older adults with diabetes attempting to live independently, especially those with cognitive impairment, which will be both effective and readily accepted.

\section{The PRIDE Project}

Providing Resources for Independence through Diabetes Education (PRIDE) was an National Institutes of Health (NIH) Planning Grant (known as an R34 grant) demonstration project funded by The National Institute of Diabetes and Digestive and Kidney Diseases (R34DK076734; C. Camp, PI), an arm of the NIH, and the first step in allowing research staff to plan, structure and execute a future, larger scale randomized control trial study. The grant was an extension of previous studies including a pilot grant from the Retirement Research Foundation (2006-079; C. Camp, PI). The project involved providing a cognitive rehabilitation intervention Spaced Retrieval (SR) to enable older adults with Type 2 diabetes and early stage dementia to remember to adhere to oral medication regimens. SR is a memory intervention that provides individuals with opportunities to recall information over progressively longer intervals of time [19]. The goal of SR is to enable individuals to remember information for clinically relevant spans of time so that they can reach long-term treatment goals. The PRIDE project also was inspired by a previous study funded by NIMH (R34 MH085246; C. Camp, PI), in which videophones were successfully used by clinical social workers to connect with HIV positive individuals with cognitive impairment to help them better adhere to their highly active antiretroviral therapy (HAART) medication regimens.

In our PRIDE project, Certified Diabetes Educators (CDEs) were connected with older adults with Type 2 diabetes and cognitive impairment (MCI or early stage dementia) living independently in their homes over the internet using tablets (iPads). Initial focus was on medication regimen adherence and an additional goal to be selected by the participant-CDE dyad (e.g., improving diet, more consistent/frequent testing of blood glucose levels, etc.). This had the advantages of delivering a Standard Education intervention program for managing Type 2 diabetes to participants in their home settings, providing live and real-time interactions between CDEs and participants, and framing the intervention as an educational program to promote independence rather than as an admission of a need for assistance. In addition, we were able to train CDEs to deliver SR during Standard Education sessions as an added component to the intervention. Thus, we could compare effects produced by education sessions alone with those produced by education session within which SR training also was used. Primary outcomes involved changes in Hemoglobin A1c (HbA1c; a measure of blood glucose levels averaged 
over two to three months) and cholesterol levels from baseline to post-intervention. Additional outcomes focused on perceived self-efficacy and depression, both of which can significantly impact health outcomes of persons living with DM.

\section{Methods}

Participants. All study participants were community dwelling and recruited using the following methods: newspaper ads, informational flyer distribution, participant referrals, and recruitment events scheduled at senior centers, senior apartment buildings, and diabetes support group meetings. Participant recruitment took place over a 16 month period. By the end of the study, the research team had responded to a total of 176 prospective participant inquiries. Final dispositions for the 176: four became pilot participants, 26 did not meet study criteria (e.g., only taking insulin, not taking oral medications, or only using diet to control blood sugar); 44 declined participation prior to enrollment; 47 screened out (19 because HbA1c was $<6.5$, 28 because they had an Telephone Interview of Cognitive Status (TICS; [20]) score > 32 (indicating no cognitive impairment), three decided to stop participation and two were dropped from the study due to extended hospitalizations. Of the remaining participants, 10 were placed on a waiting list for possible inclusion if an opening became available, and 40 completed the entire study.

Inclusion/exclusion criteria. Participants were required to be 60 years of age or older, have a diagnosis of Type 2 diabetes, be primarily responsible taking a prescribed oral diabetes medication(s), have evidence of cognitive impairment based on the TICS [20] and/or the Verabal Fluency Test (FAS; [21]—an additional measure of cognitive ability focusing on executive function) measures and have an HbA1c $>6.5$. Individuals with a diagnosis of Type 2 diabetes who were treating the condition with diet only or insulin only were excluded. Participants were paid $\$ 20.00$ for each of the 20 study related contacts (two telephone screenings, one qualifying HgA1c blood draw, two iPad orientation sessions, ten diabetes educational sessions with a Certified Diabetes Educator and five post-treatment contacts that were comprised of structured interviews and blood draws) for a maximum payment of $\$ 400.00$. A lump sum payment was made at the point the participant screened out or after the participant completed the entire study protocol. For the duration of the study involvement, participants were supplied with an iPad that had 3G/4G internet service. All participants who completed the study elected to keep the iPad. Participants took between eight and nine months to complete the entire protocol. Protocols and measures used in the PRIDE study were approved by an authorized Institutional Review Board (IRB). All recruitment and data gathering activities were conducted by research staff with extensive experience working with geriatric populations in projects funded by the NIH.

Sampling scheme. A convenience sampling methodology was used. To prevent control/treatment imbalance in this small study sample, participants were assigned to a specific treatment condition once they became eligible for the study. When attrition for one group occurred a newly enrolled participant was assigned to that treatment condition to maintain balance.

Sample description. A total of 40 participants with cognitive impairment completed the study. As described previously, cognitive impairment was measured using the TICS and the FAS. The mean TICS score was 29 (range 21 - 33) and is the equivalent of a Mini Mental Status Exam (MMSE; [22]) mean of 26 (range 20 - 28). Only one participant scored in the "non-impaired" range of TICS. With the FAS, 53\% of the sample scored in the impaired range. The mean participant age was 70 years (range 60 - 91). Females made up $80 \%$ of the sample and $82 \%$ of the participants were African American, with $18 \%$ non-Hispanic white. We recruited from senior centers in greater Cleveland, as well as public housing, which had predominant African American clientele. For educational attainment: $20 \%$ had less than a high school diploma; $50 \%$ were high school graduates; $23 \%$ had some college or a 2 year degree and $7 \%$ had an undergraduate degree. Fifty-five percent were married. Treatment condition groups, described below, were not significantly different on any of these measures.

\subsection{Design}

A between groups experimental design was used in this study to determine the extent to which strategies developed to improve self-management behaviors related to medications and blood glucose monitoring for older adults with Type 2 diabetes and early stage dementia can be learned, and under what treatment conditions (Standard Education; $\mathrm{n}=22$, versus Standard Education $+\mathrm{SR} ; \mathrm{n}=18$ ). The study also examined the feasibility 
and effectiveness of delivering the intervention via Skype on iPads.

\subsection{Procedures}

\section{Screening \& Baseline Measures; Qualifying HgA1c Test.}

Screening

The first study related contact for a prospective participant was a 45-minute telephone based screening assessment. It consisted of a study Inclusion Checklist (a 16 item assessment to ensure participant meets all the eligibility criteria), Demographic Questions (17 items), information regarding medications, FAS (inclusion criteria: $-1 \mathrm{z}$ score), and TICS (inclusion score: 21 - 32). Participants who screened out at this point were sent a check for $\$ 20.00$.

\section{Baseline}

One week later, all participants who met the TICS or FAS inclusion criteria participated in a second forty-five minute telephone baseline assessment. These included additional questions regarding medication use, current routines for managing diabetes, perceived health, and a subscale of the Multidimensional Diabetes Questionnaire (MDQ; [23]) involving perceived self-efficacy regarding management of diabetes. In addition, the short version of the Geriatric Depression Scale (GDS; [24]), a commonly used screening tool for community dwelling samples of older adults, was administered. Participants who scored $>5$ on the GDS $(n=4)$ were told to consult their physician to discuss a possible diagnosis of depression. None of these participants scored in the severely depressed range.

Qualifying HgA1c and Cholesterol Tests

The third study contact consisted of a blood draw and was scheduled at a time and place convenient to the participant. This visit took place at a participant's home with both a researcher and phlebotomist in attendance using the Bayer A1CNow test kit. If the HgA1c reading was >6.5 the individual was officially enrolled in the study and the phlebotomist did a second finger stick to obtain additional blood for the FDA approved Home Access serum cholesterol panel. Those that scored $<6.5$ on the HbA1c did not meet the study inclusion criteria were sent a check for $\$ 60.00$ to compensate them for three study contacts.

\section{iPad Orientation}

Participants were given two in-home 1-hour iPad orientation sessions. Participants were asked about current computer use and familiarity, given an overview of the use of iPads which had been configured for use by older participants with cognitive impairment and diabetes [25]. For example, the number of icons on the iPad screen was reduced to those relevant for use in the study. At the end of these sessions, participants took part in simulated Skype conversations to insure that they could use the iPads effectively during educations sessions with CDEs. Study participants are supplied with 3G/4G service for the duration of enrollment. Participants were allowed to keep their iPads at the end of participation in the study if they so desired.

\section{Educational Intervention with a Certified Diabetes Educator}

Once a participant was enrolled in the study, the participant was then assigned to either the control (Standard Education) or treatment (Standard Education + SR) condition. The educational intervention with a CDE was Skype based so the study participants did not have to leave home. The 30-minute sessions took place two times per week for approximately five weeks. Sixty percent of the participants completed all 10 sessions within the 5 week time period (mean $=5.2$ weeks); nine weeks was the longest time required by any participant.

To ensure that the CDEs implemented the 10 session intervention in a uniform and consistent manner for both the SR and control groups the project team, in collaboration with the CDEs, created a 10-session education workbook. This workbook (available from the first author on request) functioned as a detailed script that guided the CDEs through the required curriculum and data collection requirements. For both control and SR groups the CDEs worked with the participants to develop and record at least two goals related to self-management of diabetes, such as a strategies for taking medication at a specific time, regularly testing blood sugar, and monitoring carbohydrate intake. With the treatment group the CDEs had the additional requirement of using SR to help the participant master the targeted goals.

A supplemental PRIDE Study Booklet also was developed and distributed to all participants. The PRIDE Booklet is a compendium of handouts suggested for inclusion by the CDEs and addressed some of the same topics that were covered by the intervention but in greater detail, as well as additional topics not covered such as how diabetes affects the body, sick day guidelines, and guidelines for traveling with diabetes. The study participants were instructed to keep the booklet at hand during the sessions so the CDEs could refer to it for illustrative purposes. A bright and bold yellow cover made it conspicuous and easy for the participants to identify. 
Post-Intervention Follow-Up

All participants were monitored for 6 months post-treatment and had five contacts with research staff during months 1, 2, 3, 4 and 6.

Months 1 and 3 Post-Treatment, Skype Based Structured Interview: Forty-five minute structured interviews took place 4 weeks and 12 weeks after the conclusion of the education sessions. Participants were asked to recall and demonstrate the two specific goals related to diabetes management developed while working with the CDE. Self-efficacy, disease outcome expectations, and depression also were assessed at these times.

Months 2, 4, and 6 Post-Treatment Blood Draws took place 8, 16, and 24 weeks after the conclusion of the education sessions to assess A1C and serum cholesterol levels.

\section{Results}

Primary outcomes involved the HbA1C and Cholesterol Measures, shown in Table 1 and Table 2.

HbA1C. For HbA1C, data were analyzed using a mixed-model analysis of variance with the within-subject factor Time (Baseline, 2-, 4-, and 6-month follow-up), and the between-subjects factor Group (Standard Education vs Standard Education $+\mathrm{SR})$. There was a significant effect for Time, $\underline{F}(3,114)=6.85, \mathrm{p}<0.001$. Neither the Group nor the Group $\times$ Time interaction reached significance. Tests of within-subjects contrasts revealed that the Time effect was primarily a quadratic function, as seen in the total sample means at each time period. HbA1C levels declined from baseline levels after education sessions at the first two follow-ups, but returned to baseline levels at the 6-month follow-up.

Cholesterol. Cholesterol measures for Total Cholesterol, high-density lipoprotein (HDLs), low-density lipoprotein (LDLs), and Triglycerides each were analyzed using the same approach taken to analyze the HbA1C data. For Total Cholesterol, HDL, and Triglycerides, there were no significant effects. For LDL, there was a significant Group $\times$ Time interaction, $\underline{\mathrm{F}}(3,114)=4.53 ; \mathrm{p}<0.005$. In the Standard Education condition, LDL levels rose after baseline and were stable across follow-up test periods. In the Standard Education + SR group, LDL levels declined after baseline.

Secondary outcomes involved determining if trained strategies for managing diabetes were retained at followups, along with measures of self-efficacy and depression. These results are shown in Tables 3-5 respectively.

Table 1. Means (and SDs) for HBA1C Levels across the entire study.

\begin{tabular}{ccccc}
\hline & Baseline HBA1C & 2-Month FUP HBA1C & 4-Month FUP HBA1C & 6-Month FUP HBA1C \\
\hline Control $(\mathrm{n}=22)$ & $8.3(1.5)$ & $7.8(1.6)$ & $7.8(1.7)$ & $8.1(1.8)$ \\
Treatment $(\mathrm{n}=18)$ & $7.9(1.5)$ & $7.2(0.7)$ & $7.4(1.0)$ & $8.0(1.2)$ \\
OVERALL $(\mathrm{n}=40)$ & $8.1(1.5)$ & $7.5(1.3)$ & $7.6(1.4)$ & $8.1(1.6)$ \\
\hline
\end{tabular}

Table 2. Means (and SDs) for cholesterol levels across the entire study.

\begin{tabular}{ccccccccccccccccccc}
\hline & \multicolumn{4}{c}{ Baseline } & \multicolumn{4}{c}{ 2-Month FUP } & \multicolumn{4}{c}{ 4-Month FUP } & \multicolumn{4}{c}{ 6-Month FUP } \\
\hline & TC & HDL & LDL & TG & TC & HDL & LDL & TG & TC & HDL & LDL & TG & TC & HDL & LDL & TG \\
\hline $\begin{array}{c}\text { Control } \\
\text { (n= 22) }\end{array}$ & 155 & 51 & 75 & 143 & 171 & 56 & 91 & 123 & 171 & 55 & 90 & 128 & 166 & 52 & 89 & 126 \\
Treatment & 155 & 51 & 82 & 115 & 153 & 51 & 80 & 109 & 146 & 50 & 71 & 130 & 150 & 51 & 74 & 126 \\
$(\mathbf{n = 1 8 )}$ & $(31)$ & $(13)$ & $(23)$ & $(43)$ & $(31)$ & $(11)$ & $(22)$ & $(48)$ & $(22)$ & $(12)$ & $(18)$ & $(70)$ & $(24)$ & $(12)$ & $(18)$ & $(50)$ \\
OVERALL & 155 & 51 & 78 & 130 & 163 & 54 & 86 & 116 & 160 & 53 & 82 & 129 & 158 & 52 & 83 & 126 \\
$(\mathbf{n = 4 0 )}$ & $(43)$ & $(15)$ & $(30)$ & $(76)$ & $(33)$ & $(13)$ & $(24)$ & $(58)$ & $(33)$ & $(12)$ & $(29)$ & $(64)$ & $(30)$ & $(12)$ & $(23)$ & $(55)$ \\
\hline
\end{tabular}

Table 3. Strategy usage at 1- and 3-Month follow-ups.

\begin{tabular}{ccc}
\hline & 1-Month FUP & 3-Month FUP \\
\hline Control $(\mathrm{n}=22)$ & $82 \%$ & $82 \%$ \\
Treatment $(\mathrm{n}=18)$ & $100 \%$ & $89 \%$ \\
OVERALL $(\mathrm{n}=40)$ & $90 \%$ & $85 \%$ \\
\hline
\end{tabular}


Table 4. Means (and SDs) for multi-dimensional diabetes questionnaire-self efficacy sub scores—at 1- and 3-Month follow-ups.

\begin{tabular}{cccc}
\hline & Baseline & 1-Month FUP & 3-Month FUP \\
\hline Control $(\mathrm{n}=22)$ & $8.5(3.1)$ & $10.3(2.3)$ & $10.5(2.0)$ \\
Treatment $(\mathrm{n}=18)$ & $8.7(3.1)$ & $11.6(2.3)$ & $11.3(2.1)$ \\
OVERALL $(\mathrm{n}=40)$ & $8.6(3.1)$ & $10.9(2.3)$ & $10.9(2.1)$ \\
\hline
\end{tabular}

Table 5. Means (and SDs) for geriatric depression scores.

\begin{tabular}{cccc}
\hline & Baseline & 1-Month FUP & 3-Month FUP \\
\hline Control $(\mathrm{n}=22)$ & $1.8(1.9)$ & $1.9(2.1)$ & $1.7(1.8)$ \\
Treatment $(\mathrm{n}=18)$ & $2.7(2.7)$ & $1.7(2.3)$ & $2.0(2.8)$ \\
OVERALL $(\mathrm{n}=40)$ & $2.2(2.3)$ & $1.8(2.2)$ & $1.9(2.3)$
\end{tabular}

Strategies used. Use of strategies to manage diabetes was assessed at 1- and 3-month follow-ups. In the Standard Education group, $82 \%$ of participants were using trained strategies at both follow-ups. In the Standard Education + SR group, 100\% were using trained strategies at the 1-month follow-up, and 89\% were still using trained strategies at the 3-month follow-up.

MDQ Self-Efficacy Subtest. For the self-efficacy subtest of the MDQ, measures were taken at baseline and at 1-and 3-month follow-ups. Data for this subtest were analyzed using a mixed-model analysis of variance with the within-subject factor Time (Baseline, 1-, and 3-month follow-up), and the between-subjects factor Group (Standard Education vs Standard Education + SR).

For self-efficacy, there was a significant effect for Time, $\underline{F}(2,76)=20.84 ; p<0.001$. Self-efficacy in participants significantly increased after baseline and was maintained across follow-ups.

Depression. Depression was assessed at the same times as the MDQ self-efficacy subtest, and analyzed in a similar way. No effects were significant, with overall levels of depression remaining low throughout the course of the study.

\section{Conclusions}

We found that for HbA1C, a measure of average blood sugar level, there was a significant decline in blood sugar from baseline to the first two post-test intervals, but that there was return to baseline levels at the 6-month follow-up assessment. Given that our design did not call for continual contact between participants and our CDEs, this is not an entirely surprising finding, and reinforces the need to maintain connection between clinicians and persons with Type 2 diabetes and cognitive impairment. The ability to maintain connection between interventionists and participants during their training sessions using iPads and the internet suggests that this approach lends itself to long-term connection and maintenance programs for persons with diabetes and clinicians. CDEs found the iPads easy to use and that the face-to-face nature of their interactions with participants was reported to be of benefit in establishing good clinical rapport with participants.

There was no significant difference between the two comparison groups on HbA1C measures, which may be explained by the high number of strategies used to manage diabetes that were retained at follow-ups under both conditions, though the group which also received SR had a trend towards better retention of strategies. Our CDEs were effective in both teaching and maintaining strategy use in the short term, had prior experience with clients' challenges in remembering to take medications, and provided strategies for helping be more consistent with medication adherence. CDEs were not prohibited from using this part of their standard procedures in the current study, even if they were working with persons in the control condition. Therefore, this could have diluted the effects of the SR intervention.

Again, long-term maintenance probably will involve extended contact between persons with diabetes and clinicians.

Participants showed an increased sense of self-efficacy after working with CDEs, an outcome which supports 
the idea that the interactions between these dyads were positive and viewed as helpful by participants. In addition, only $2 \%$ of participants reported that the iPad was difficult to use, and $95 \%$ reported that they planned to continue using the iPad after the conclusion of the study. Planned uses included social media, online banking, games, internet, camera, communication [by email], music, looking up information (e.g., health), using apps, using Skype with friends, tutoring school children, using apps for reading and math, reading books, and using maps. This extensive list of activities suggested that the respondents were well instructed on the iPad's capabilities during their two orientation sessions and now are enthusiastic users of on-line technology. This also bodes well for the potential of this approach in connecting persons with Type 2 diabetes and cognitive impairments with clinical services in their homes.

Finally, most comparisons of the two treatment conditions were not significant. However, we did have the intriguing interaction between group and time for LDL cholesterol levels. As mentioned previously, in the Standard Education + SR group LDL levels declined after baseline while LDL levels rose after baseline in the Standard Education alone condition. While intriguing, we need to better understand why this outcome is obtained and to determine whether this effect is reliable in future studies. At the least, it encourages us to continue using SR and to compare its effects with standard educational programs.

Future studies should involve working with a larger and more diverse sample within the context of a randomized control trial, providing booster sessions after initial training, and possibly using peer volunteers to help maintain contact over extended time frames in a cost-effective manner. This latter approach has shown promise in persons with severe mental illness and comorbid diabetes [26], and could be adapted to the current intervention approach.

\section{References}

[1] Ogden, C.L., Carroll, M.D., Kit, B.K. and Flegal, K.M. (2014) Prevalence of Childhood and Adult Obesity in the United States, 2011-2012. JAMA, 311, 806-814. http://dx.doi.org/10.1001/jama.2014.732

[2] Centers for Disease Control and Prevention (2014) National Diabetes Statistics Report: Estimates of Diabetes and Its Burden in the United States, 2014. US Department of Health and Human Services, Atlanta.

[3] National Diabetes Fact Sheet (2011) http://www.cdc.gov/diabetes/pubs/pdf/ndfs 2011.pdf

[4] Wang, Y.C., McPherson, K., Marsh, T., Gortmaker, S.L. and Brown, M. (2011) Health and Economic Burden of the Projected Obesity Trends in the USA and the UK. The Lancet, 378, 815-825. http://dx.doi.org/10.1016/S0140-6736(11)60814-3

[5] Zhang, P., Zhang, X., Brown, J., Vistisen, D., Sicree, R., Shaw, J. and Nichols, G. (2010) Global Healthcare Expenditure on Diabetes for 2010 and 2030. Diabetes Research and Clinical Practice, 87, 293-301. http://dx.doi.org/10.1016/j.diabres.2010.01.026

[6] Känel, R., Dimsdale, J.E., et al. (2006) Poor Sleep Is Associated with Higher Plasma Proinflammatory Cytokine Interleukin-6 and Procoagulant Marker Fibrin D-Dimer in Older Caregivers of People with Alzheimer's Disease. Journal of the American Geriatrics Society, 54, 431-437. http://dx.doi.org/10.1111/j.1532-5415.2005.00642.x

[7] Vitaliano, P.P., Russo, J., Scanlan, J.M. and Greeno, C.G. (1996) Weight Changes in Caregivers of Alzheimer's Care Recipients: Psychobehavioral Predictors. Psychology and Aging, 11, 155.

http://dx.doi.org/10.1037/0882-7974.11.1.155

[8] Shaw, W.S., Patterson, T.L., Ziegler, M.G., Dimsdale, J.E., Semple, S.J. and Grant, I. (1999) Accelerated Risk of Hypertensive Blood Pressure Recordings among Alzheimer Caregivers. Journal of Psychosomatic Research, 46, 215227. http://dx.doi.org/10.1016/S0022-3999(98)00084-1

[9] Lee, S., Colditz, G.A., Berkman, L.F. and Kawachi, I. (2003) Caregiving and Risk of Coronary Heart Disease in US Women: A Prospective Study. American Journal of Preventive Medicine, 24, 113-119. http://dx.doi.org/10.1016/S0749-3797(02)00582-2

[10] Alzheimer's Association (2012) 2012 Alzheimer’s Disease Facts and Figures. http://www.alz.org/downloads/facts_figures_2012.pdf

[11] Stewart, R. and Liolitsa, D. (1999) Type 2 Diabetes Mellitus, Cognitive Impairment and Dementia. Diabetic Medicine, 16, 93-112. http://dx.doi.org/10.1046/j.1464-5491.1999.00027.x

[12] Coker, L.H. and Shumaker, S.A. (2003) Type 2 Diabetes Mellitus and Cognition: An Understudied Issues in Women’s Health. Journal of Psychosomatic Research, 54, 129-139. http://dx.doi.org/10.1016/S0022-3999(02)00523-8

[13] Ryan, C.M. and Geckle, M. (2000) Why Is Learning and Memory Dysfunction in Type 2 Diabetes Limited to Older Adults? Diabetes/Metabolism Research and Reviews, 16, 308-315. 
http://dx.doi.org/10.1002/1520-7560(2000)9999:9999<::AID-DMRR141>3.0.CO;2-X

[14] Haan, M. (2009) Type 2 Diabetes and Incidence of Dementia and Cognitive Decline: What Are the Mechanisms? Alzheimer's \& Dementia: The Journal of the Alzheimer's Association, 5, 123.

[15] Anderson, R.J., Freedland, K.E., Clouse, R.E. and Lustman, P.J. (2001) The Prevalence of Comorbid Depression in Adults with Diabetes: A Meta-Analysis. Diabetes Care, 24, 1069-1078. http://dx.doi.org/10.2337/diacare.24.6.1069

[16] Lin, E.H., Katon, W., Von Korff, M., Rutter, C., Simon, G.E., Oliver, M., Young, B., et al. (2004) Relationship of Depression and Diabetes Self-Care, Medication Adherence, and Preventive Care. Diabetes Care, 27, 2154-2160. http://dx.doi.org/10.2337/diacare.27.9.2154

[17] Ciechanowski, P.S., Katon, W.J. and Russo, J.E. (2000) Depression and Diabetes: Impact of Depressive Symptoms on Adherence, Function, and Costs. JAMA Internal Medicine, 160, 3278-3285. http://dx.doi.org/10.1001/archinte.160.21.3278

[18] Alzheimer's Association (2014) 2013 Alzheimer’s Disease Facts and Figures. http://www.alz.org/downloads/facts_figures_2013.pdf

[19] Camp, C.J. (2006) Spaced Retrieval: A Case Study in Dissemination of a Cognitive Intervention for Persons with Dementia. In: Attix, D.K. and Welsch-Bohmner, K.A., Eds., Geriatric Neuropsychological Assessment and Intervention, The Guilford Press, New York, 275-292.

[20] Brandt, J., Spencer, M. and Folstein, M. (1988) The Telephone Interview for Cognitive Status. Neuropsychiatry, Neuropsychology, \& Behavioral Neurology, 1, 111-117.

[21] Spreen, O. and Strauss, E. (1998) A Compendium of Neuropsychological Tests. Oxford University Press, New York, 447-459.

[22] Folstein, M.F., Folstein, S.E. and McHugh, P.R. (1975) "Mini-Mental State”. A Practical Method for Grading the Cognitive State of Patients for the Clinician. Journal of Psychiatric Research, 12, 189-198. http://dx.doi.org/10.1016/0022-3956(75)90026-6

[23] Talbot, F., Nouwen, A., Gingras, J., Gosselin, M. and Audent, J. (1997) The Assessment of Diabetes-Related Cognitive and Social Factors: The Multidimensional Diabetes Questionnaire. Journal of Behavioral Medicine, 20, $291-312$. http://dx.doi.org/10.1023/A:1025508928696

[24] Herrmann, N., Mitimann, N., Silver, I.L., Shulman, K.L., Busto, U., Shear, N.H. and Naranjo, C.A. (1996) A Validation Study of the Geriatric Depression Scale Short Form. International Journal of Geriatric Psychiatry, 11, 457-460. http://dx.doi.org/10.1002/(SICI)1099-1166(199605)11:5<457::AID-GPS325>3.0.CO;2-2

[25] Camp, C.J., Skrajner, M.J., Antenucci, V., Frentiu, C. and Haberman, J. (2012) Creating Effective Self-Management for Older Adults with Type 2 Diabetes and Memory Impairment. Gerontologist, 52, 130.

[26] Sajatovic, M., Dawson, N.V., Perzynski, A., Blixen, C., Bialko, C., McKibbin, C., Bauer, M.S., Seeholzer, E., Kaiser, D. and Fuentes-Casiano, E. (2011) Optimizing Care for People with Serious Mental Illness and Comorbid Diabetes. Psychiatric Services (Best Practices Column), 62, 1001-1003. http://dx.doi.org/10.1176/ps.62.9.pss6209 1001 